\title{
Vorwort \& Dank zur 2. Auflage
}

Der gute Zuspruch zur ersten Auflage, die ermutigende Resonanz der Fachleserschaft sowie der Wunsch vieler Leser nach einer elektronischen Version hat diese zweite Auf lage hervorgebracht.

Frau Karin Sora, Vice president Science, Technology, Engineering \& Mathematics (STEM), vom Verlagshaus DeGruyter (DG) danke ich sehr herzlich für ihr Interesse dieses Buch zu produzieren und herauszugeben.

Frau Dr. Carina Kniep, ehemals Editorial Director Physical Sciences/DG, danke ich für die wertvolle Koordination in der Frühphase dieses Projekts.

Frau Lena Stoll, Project Editor Chemistry \& Materials Science/DG und Frau Dr. Ria Fritz, Project Editor Chemistry/DG, danke ich für die sehr gute Zusammenarbeit bei der Konzeption, Umsetzung und Werbung für dieses Buch.

Frau Jeannette Krause, Verlagsherstellung/le-tex publishing services $\mathrm{GmbH}$, danke ich sehr für die stets rasche und reibungslose Kommunikation sowie die umsichtige und gelungene Umsetzung eines komplizierten Manuskripts.

Diese zweite, korrigierte Auflage ist gegenüber der ersten Auflage um 98 neue Stichworte, zahlreiche weitere Literaturstellen und Tabellen erweitert. Außerdem ist sie nun auch als elektronische Version (E-Book) verfügbar.

Ich bedanke mich erneut bei vielen Kolleginnen und Kollegen für ihre Hinweise und Korrekturen. Insbesondere danken möchte ich:

Herrn Dr. Werner Arnold, Ingolstadt, für die kritische Durchsicht, Korrektur und Ergänzung vieler Stichworte im Bereich Detonik und Panzerdurchschlag,

Herrn Dr. Kurt Schubert, Gunzenhausen, für die akribische Durchsicht der ersten Auflage nach sachlichen und Rechtschreibfehlern, und

Herrn Dr. Paul Wanninger, Adelsberg, für seine gewissenhafte Durchsicht, kritische Rezension der ersten Auflage sowie für viele Hinweise zu Fehlern und fehlenden Einträgen und die Überlassung umfangreicher Informationen zur Technologie in Deutschland entwickelter kunststoffgebundener Explosivstoffe.

Ich wünsche Ihnen nun mit diesem Buch erfolgreiche Recherchen sowie wertvolle Impulse für Ihre Arbeit.

Weiterhin würde ich mich sehr freuen, Hinweise auf Fehler und notwendige Ergänzungen für zukünftige Auflagen dieses Buches zu erhalten.

Kaiserslautern im April 2019

Ernst-Christian Koch 